\title{
Relativistic allowed and forbidden transition probabilities for fluorine-like Fe XVIII ${ }^{\star}$
}

\author{
V. Jonauskas ${ }^{1, \star \star}$, F. P. Keenan ${ }^{1}$, M. E. Foord ${ }^{2}$, R. F. Heeter ${ }^{2}$, S. J. Rose ${ }^{3}$, P. A. M. van Hoof $^{1}$, \\ G. J. Ferland ${ }^{4}$, K. M. Aggarwal ${ }^{1}$, R. Kisielius ${ }^{1}$, and P. H. Norrington ${ }^{5}$ \\ 1 Department of Pure and Applied Physics, The Queen's University of Belfast, Belfast BT7 1NN, Northern Ireland, UK \\ 2 University of California, Lawrence Livermore National Laboratory, Livermore, CA 94551, USA \\ 3 Department of Physics, Clarendon Laboratory, Parks Road, Oxford OX1 3PU, UK \\ ${ }^{4}$ Department of Physics, University of Kentucky, Lexington, KY 40506, USA \\ 5 Department of Applied Mathematics and Theoretical Physics, The Queen's University of Belfast, Belfast BT7 1NN, \\ Northern Ireland, UK
}

Received 17 September 2003 / Accepted 19 November 2003

\begin{abstract}
Energy levels and the corresponding transition probabilities for allowed and forbidden transitions among the levels of the ground configuration and first 23 excited configurations of fluorine-like Fe XVIII have been calculated using the multiconfigurational Dirac-Fock GRASP code. A total of 379 lowest bound levels of Fe XVIII is presented, and the energy levels are identified in spectroscopic notations. Transition probabilities, oscillator strengths and line strengths for electric dipole (E1), electric quadrupole (E2) and magnetic dipole (M1) transitions among these 379 levels are also presented. The calculated energy levels and transition probabilities are compared with experimental data.
\end{abstract}

Key words. atomic data

\section{Introduction}

An interest in atomic data for F-like Fe XVIII arises from the X-ray spectra of astrophysical sources obtained from satellite-borne observatories, such as the NASA Chandra $X$ ray Observatory and the ESA XMM-Newton. In order to analyse the huge amount of observations from these missions, it is necessary to have accurate atomic data. Several papers have been published concerning energy level and radiative rate calculations for F-like Fe. Bromage et al. (1977) calculate wavelengths and oscillator strengths of spectral lines belonging to the $1 s^{2} 2 s^{2} 2 p^{5}-1 s^{2} 2 s^{2} 2 p^{4} 4 d$ transition array. Fawcett (1984) provide energy levels, wavelengths and oscillator strengths calculated by the HXR method of Cowan (1981). The ab initio values of Slater radial integrals are optimized on the basis of observed energy levels and wavelengths in their calculations. Sampson et al. (1991) present oscillator strengths and transition energies as well as collision strengths obtained by their relativistic code for $1 \mathrm{~s}^{2} 2 \mathrm{~s}^{2} 2 \mathrm{p}^{5}, 1 \mathrm{~s}^{2} 2 \mathrm{~s}^{1} 2 \mathrm{p}^{6}, 1 \mathrm{~s}^{2} 2 \mathrm{~s}^{2} 2 \mathrm{p}^{4} 3 l$, $1 \mathrm{~s}^{2} 2 \mathrm{~s}^{1} 2 \mathrm{p}^{5} 3 l$ and $1 \mathrm{~s}^{2} 2 \mathrm{p}^{6} 3 l \quad(l=0,1,2)$ configurations, corresponding to 113 levels. Cornille et al. (1992) calculate

Send offprint requests to: V. Jonauskas,

e-mail: v.jonauskas@qub.ac.uk

* Tables 5 to 8 are only available in electronic form at the CDS via anonymous ftp to cdsarc.u-strasbg.fr $(130.79 .128 .5)$ or via http://cdsweb.u-strasbg.fr/cgi-bin/qcat?]/A+A/416/383

$\star \star$ On leave from: Vilnius University Institute of Theoretical Physics and Astronomy, A. Goštauto 12, 2600 Vilnius, Lithuania. energies for the 108 lowest levels, and also wavelengths and radiative transition probabilities using the SUPERSCTRUCTURE program (Eissner et al. 1974). Blackford \& Hibbert (1994) employ the CIV3 code of Hibbert \& Glass (1978) for oscillator strengths among the states belonging to the $1 s^{2} 2 s^{2} 2 p^{5}$, $1 s^{2} 2 s^{1} 2 p^{6}$ and $1 s^{2} 2 s^{2} 2 p^{4} 3 l$ configurations, while Palmeri et al. (2003) calculate energy levels, radiative and Auger rates for K-vacancy states with AUTOSTRUCTURE (Badnell 1986, 1997), the Cowan code (Cowan 1981) and Breit-Pauli $R$-matrix suite (Burke et al. 1971).

All of the above calculations contain smaller sets of energy levels and transitions than considered in the present work. In our calculations we employ a larger CI basis than other authors and relativistic Dirac-Fock functions. A large basis of interacting configurations enables us to improve atomic state functions and leads to more reliable energy levels and transition data, as we show below. The 379 lowest energy levels of Fe XVIII are considered, and transition probabilities as well as oscillator strengths among these levels are calculated. A comparison is made between experimental data and our calculations, as well as the theoretical results obtained by Sampson et al. (1991) and Cornille et al. (1992).

\section{Calculations}

We are using the GRASP code of Dyall et al. (1989), in which the relativistic corrections which correspond in the 
non-relativistic limit to mass, Darwin, spin-orbit and a part of spin-other-orbit corrections (Armstrong 1966; Karazija \& Jonauskas 2001) are included in one-electron wave functions by solving multiconfigurational Dirac-Fock (MCDF) equations. MCDF, compared with the conventional non-relativistic Breit-Pauli approximation, enables one to introduce direct and indirect relativistic effects into wave functions. This is important, when the contraction of inner orbitals leads to more effective screening of the nucleus, and pushes valence orbitals away from the nucleus. The Breit and QED corrections are considered in the first order of perturbation theory, and the correlation corrections are taken into account by the configuration interaction (CI) technique.

We report results for the 379 lowest energy levels of Fe XVIII, originating from $1 \mathrm{~s}^{2} 2 \mathrm{~s}^{2} 2 \mathrm{p}^{5}, 1 \mathrm{~s}^{2} 2 \mathrm{~s}^{1} 2 \mathrm{p}^{6}, 1 \mathrm{~s}^{2} 2 \mathrm{~s}^{2} 2 \mathrm{p}^{4} 3 l$, $1 \mathrm{~s}^{2} 2 \mathrm{~s}^{2} 2 \mathrm{p}^{4} 4 l^{\prime}, \quad 1 \mathrm{~s}^{2} 2 \mathrm{~s}^{2} 2 \mathrm{p}^{4} 5 l^{\prime \prime}, \quad 1 \mathrm{~s}^{2} 2 \mathrm{p}^{6} 3 l, \quad 1 \mathrm{~s}^{2} 2 \mathrm{~s}^{1} 2 \mathrm{p}^{5} 3 l$, $1 \mathrm{~s}^{2} 2 \mathrm{~s}^{1} 2 \mathrm{p}^{5} 4 l^{\prime}$ and $1 \mathrm{~s}^{2} 2 \mathrm{~s}^{2} 2 \mathrm{p}^{4} 6 \mathrm{~s}\left(l=0,1,2, l^{\prime}=0,1,2,3\right.$ and $\left.l^{\prime \prime}=0,1,2,3,4\right)$ configurations. The highest level of the $1 s^{2} 2 s^{2} 2 p^{4} 5 g$ configuration is chosen as the highest level for the presented data. Hence some levels of the $1 s^{2} 2 s^{1} 2 p^{5} 4 d$, $1 s^{2} 2 s^{1} 2 p^{5} 4 f$ and $1 s^{2} 2 s^{2} 2 p^{4} 6 s$ configurations are not provided in this work, as their binding energies are smaller than our selected cut-off value.

The CI basis for the generation of atomic state functions additionally includes one-electron excitations from $2 \mathrm{~s}$ and $2 \mathrm{p}$ orbitals of the $1 \mathrm{~s}^{2} 2 \mathrm{~s}^{2} 2 \mathrm{p}^{5}$ configuration until the $7 \mathrm{i}$ orbital, twoelectron excitations from $2 \mathrm{~s}$ or/and $2 \mathrm{p}$ orbitals to all possible combinations of two electrons in the shells with $n=3$. The level energies and atomic state functions obtained after the diagonalisation of the Hamiltonian matrix are used to evaluate the transition probabilities, oscillator and line strengths of electric dipole (E1), electric quadrupole (E2) and magnetic dipole (M1) type transitions between the states of all the above mentioned configurations. The two strongest and the total radiative transition probabilities from the level are also obtained. To our knowledge, our work represents the largest calculation to date for Fe XVIII.

\section{Results and discussion}

The calculated energies of the 107 levels of $1 \mathrm{~s}^{2} 2 \mathrm{~s}^{1} 2 \mathrm{p}^{6}$, $1 \mathrm{~s}^{2} 2 \mathrm{~s}^{2} 2 \mathrm{p}^{4} 3 l$ and $1 \mathrm{~s}^{2} 2 \mathrm{~s}^{1} 2 \mathrm{p}^{5} 3 l$ configurations with respect to the ground $1 \mathrm{~s}^{2} 2 \mathrm{~s}^{2} 2 \mathrm{p}^{5}$ configuration are shown in Table 1. Other calculated 271 energy levels (Table 5) belonging to $1 \mathrm{~s}^{2} 2 \mathrm{~s}^{2} 2 \mathrm{p}^{4} 4 l^{\prime}, \quad 1 \mathrm{~s}^{2} 2 \mathrm{~s}^{2} 2 \mathrm{p}^{4} 5 l^{\prime \prime}, \quad 1 \mathrm{~s}^{2} 2 \mathrm{p}^{6} 3 l, 1 \mathrm{~s}^{2} 2 \mathrm{~s}^{1} 2 \mathrm{p}^{5} 4 l^{\prime}$ and $1 \mathrm{~s}^{2} 2 \mathrm{~s}^{2} 2 \mathrm{p}^{4} 6 \mathrm{~s}\left(l=0,1,2, l^{\prime}=0,1,2,3\right.$ and $\left.l^{\prime \prime}=0,1,2,3,4\right)$ configurations are available in electronic form at the CDS. The two lowest levels of the $1 s^{2} 2 s^{2} 2 p^{4} 6 s$ configuration are presented since their binding energies are larger then those of the highest level of the $1 s^{2} 2 s^{2} 2 p^{4} 5 g$ configuration. Due to this some levels of the $1 s^{2} 2 s^{1} 2 p^{5} 4 d$ and $1 s^{2} 2 s^{1} 2 p^{5} 4 f$ configurations are not presented in the paper. The first column of Table 1 provides an index for the corresponding level, while configurations are presented in the second column using the jj coupling scheme. For brevity, filled $1 s_{1 / 2}$ and $2 s_{1 / 2}$ subshells are omitted in the notation of configuration. Intermediate many electron quantum numbers are shown in the parentheses.
In the third column of Table 1 the energy levels are identified using LSJ spectroscopic notations. The spectroscopic notation corresponds to the configuration state function with the primary contribution in atomic state function obtained in the LS coupling scheme. In some cases at least two levels can have the same identification. If this occurs, the superscript in the parentheses to the term notation marks the index of the lowest level which has the same LSJ characteristic.

To ensure the completeness of the spectroscopic dataset, the reclassification of terms in the level notations in Table 2 is proposed, although the identification of levels in the Table 1 is used through the paper. This is organised as follows. If two levels have the same many electron quantum numbers for a given configuration in Table 1, the identification of the level with the smaller contribution to the primary atomic state function is changed to that of the secondary configuration state function. If this secondary function is employed for the identification of some other level, then the third configuration state function is checked.

The two major radiative transition probabilities from each level, as well as the total radiative transition probability (important to obtain the lifetime of a level and the branching ratio) are presented in Table 1. These transition probabilities include the sum of all E1, E2 and M1 results from the level.

Our energy levels are compared with the experimental values compiled by NIST (National Institute of Standards and Technology: www . physics .nist.gov/PhysRefData) in Table 3 . The greatest deviation of $1.4 \%$ from the experimental value is obtained for the $2 \mathrm{~s} 2 \mathrm{p}^{6}{ }^{2} \mathrm{~S}_{1 / 2}$ level. However the Cornille et al. (1992) value of $1081168 \mathrm{~cm}^{-1}$ is higher than our result of $1079594 \mathrm{~cm}^{-1}$, while Sampson et al. (1991) provide a larger estimate $\left(1088033 \mathrm{~cm}^{-1}\right)$. Palmeri et al. (2003) obtain an energy of $1064724 \mathrm{~cm}^{-1}$ for that level. This is closer to the experimental value, but they use improved relativistic wavefunctions obtained in terms of nonrelativistic functions with LS term energy differences adjusted to fit the centres of gravity of the experimental multiplet. Hence their value is not obtained using ab initio calculations, and cannot really be compared with our data. The average deviation between our energy levels and the experimental values is only $0.2 \%$, and does not exceed $0.7 \%$, except for the above mentioned $2 \mathrm{~s} 2 \mathrm{p}^{6}{ }^{2} \mathrm{~S}_{1 / 2}$ level.

Multiconfigurational wavefunctions obtained after the diagonalisation of the Hamiltonian matrix are used to compute matrix elements of the electric and magnetic transition operators (electric operators are considered in the Coulomb and Babushkin gauges), and later applied for the calculation of transition probabilities, line and oscillator strengths of E1, E2 and M1 transitions. The total number of dipole allowed and intercombination E1-type transitions is 20742, while the complete set of forbidden transitions totals 48330. Table 6 (Tables 6-8 are only available in electronic form at the CDS) includes only transitions for which oscillator strengths exceed 0.001. Forbidden E2-type transitions for which f-values are larger than $10^{-6}$ are presented in Table 7 . Table 8 provides data for M1-type transitions with f-values exceeding $10^{-7}$.

The fairly good agreement between our calculated transition probabilities and the NIST data is illustrated in Table 4. An average deviation from the experimental values of $10 \%$ is 
Table 1. Calculated energy levels of Fe XVIII (in $\mathrm{cm}^{-1}$ ) relative to the ground energy with spectroscopic notations. The two largest spontaneous radiative transition probabilities $A^{r}$ (in s${ }^{-1}$ ) from each level are given. Arrows indicate the final level to which radiative transition happens from the level. The sum of all radiative probabilities from the initial level is given in the last column. The term notation superscript number in parentheses marks the lowest level with the same spectroscopic identification. Note that $a(b) \equiv a \times 10^{b}$.

\begin{tabular}{|c|c|c|c|c|c|c|c|c|}
\hline Index & $\begin{array}{l}\text { Configuration } \\
\text { (jj scheme) }\end{array}$ & LSJ & $E\left(\mathrm{~cm}^{-1}\right)$ & $\begin{array}{l}\text { final } \\
\text { level }\end{array}$ & $A^{r}\left(\mathrm{~s}^{-1}\right)$ & $\begin{array}{l}\text { final } \\
\text { level }\end{array}$ & $A^{r}\left(\mathrm{~s}^{-1}\right)$ & $\sum A^{r}\left(\mathrm{~s}^{-1}\right)$ \\
\hline 1 & $2 p_{1 / 2}^{2} 2 p_{3 / 2}^{3}$ & ${ }^{2} \mathrm{P}_{3 / 2}$ & -241722494. & & & & & \\
\hline 2 & $2 \mathrm{p}_{1 / 2}^{1} 2 \mathrm{p}_{3 / 2}^{4}$ & ${ }^{2} \mathrm{P}_{1 / 2}$ & 102063. & $\rightarrow 1$ & $1.905(04)$ & & & $1.905(04)$ \\
\hline 3 & $2 \mathrm{~s}_{1 / 2}^{1} 2 \mathrm{p}_{1 / 2}^{2} 2 \mathrm{p}_{3 / 2}^{4}$ & ${ }^{2} \mathrm{~S}_{1 / 2}$ & 1079594. & $\rightarrow 1$ & $8.313(10)$ & $\rightarrow 2$ & $3.035(10)$ & $1.135(11)$ \\
\hline 4 & $2 \mathrm{p}_{1 / 2}^{2} 2 \mathrm{p}_{3 / 2}^{2}(2.0) 3 \mathrm{~s}_{1 / 2}^{1}$ & ${ }^{4} \mathrm{P}_{5 / 2}$ & 6206772. & $\rightarrow 1$ & $8.312(10)$ & $\rightarrow 3$ & $2.354(05)$ & $8.312(10)$ \\
\hline 5 & $2 \mathrm{p}_{1 / 2}^{2} 2 \mathrm{p}_{3 / 2}^{2}(2.0) 3 \mathrm{~s}_{1 / 2}^{1}$ & ${ }^{2} \mathrm{P}_{3 / 2}$ & 6234827. & $\rightarrow 1$ & $1.651(12)$ & $\rightarrow 2$ & $4.336(10)$ & $1.694(12)$ \\
\hline 6 & $2 \mathrm{p}_{1 / 2}^{2} 2 \mathrm{p}_{3 / 2}^{2}(0.0) 3 \mathrm{~s}_{1 / 2}^{1}$ & ${ }^{4} \mathrm{P}_{1 / 2}$ & 6285591. & $\rightarrow 1$ & $1.703(11)$ & $\rightarrow 2$ & 4.917(09) & $1.752(11)$ \\
\hline 7 & $2 \mathrm{p}_{1 / 2}^{1} 2 \mathrm{p}_{3 / 2}^{3}(1.0) 3 \mathrm{~s}_{1 / 2}^{1}$ & ${ }^{4} \mathrm{P}_{3 / 2}$ & 6303529. & $\rightarrow 1$ & $8.597(11)$ & $\rightarrow 2$ & $7.100(10)$ & $9.307(11)$ \\
\hline 8 & $2 \mathrm{p}_{1 / 2}^{1} 2 \mathrm{p}_{3 / 2}^{3}(1.0) 3 \mathrm{~s}_{1 / 2}^{1}$ & ${ }^{2} \mathrm{P}_{1 / 2}$ & 6329621. & $\rightarrow 2$ & $1.399(12)$ & $\rightarrow 1$ & $1.234(12)$ & $2.633(12)$ \\
\hline 9 & $2 \mathrm{p}_{1 / 2}^{1} 2 \mathrm{p}_{3 / 2}^{3}(2.0) 3 \mathrm{~s}_{1 / 2}^{1}$ & ${ }^{2} \mathrm{D}_{5 / 2}$ & 6387985. & $\rightarrow 1$ & $1.021(12)$ & $\rightarrow 3$ & $3.055(06)$ & $1.021(12)$ \\
\hline 10 & $2 \mathrm{p}_{1 / 2}^{1} 2 \mathrm{p}_{3 / 2}^{3}$ & ${ }^{2} \mathrm{D}_{3 / 2}$ & 6391884. & $\rightarrow 2$ & $1.240(12)$ & $\rightarrow 1$ & $1.799(10)$ & $1.258(12)$ \\
\hline 11 & $2 \mathrm{p}_{1 / 2}^{2} 2 \mathrm{p}_{3 / 2}^{2}(2.0) 3 \mathrm{p}_{1 / 2}^{1}$ & ${ }^{4} \mathrm{P}_{3 / 2}$ & 6452996. & $\rightarrow 4$ & 2.757(09) & $\rightarrow 3$ & $1.221(09)$ & 4.601(09) \\
\hline 12 & $2 \mathrm{p}_{1 / 2}^{2} 2 \mathrm{p}_{3 / 2}^{2}(2.0) 3 \mathrm{p}_{1 / 2}^{1}$ & ${ }^{4} \mathrm{P}_{5 / 2}$ & 6456313. & $\rightarrow 4$ & $3.486(09)$ & $\rightarrow 1$ & $1.216(08)$ & $3.653(09)$ \\
\hline 13 & $2 \mathrm{p}_{1 / 2}^{2} 2 \mathrm{p}_{3 / 2}^{2}(2.0) 3 \mathrm{p}_{3 / 2}^{1}$ & ${ }^{4} \mathrm{P}_{1 / 2}^{(19)}$ & 6484099. & $\rightarrow 5$ & $3.055(09)$ & $\rightarrow 3$ & $1.825(09)$ & $5.589(09)$ \\
\hline 14 & $2 \mathrm{p}_{1 / 2}^{2} 2 \mathrm{p}_{3 / 2}^{2}(2.0) 3 \mathrm{p}_{3 / 2}^{1}$ & ${ }^{4} \mathrm{D}_{7 / 2}$ & 6489627. & $\rightarrow 4$ & $5.570(09)$ & $\rightarrow 1$ & $1.029(08)$ & $5.673(09)$ \\
\hline 15 & $2 \mathrm{p}_{1 / 2}^{2} 2 \mathrm{p}_{3 / 2}^{2}(2.0) 3 \mathrm{p}_{3 / 2}^{1}$ & ${ }^{2} \mathrm{D}_{5 / 2}$ & 6490723. & $\rightarrow 5$ & $4.006(09)$ & $\rightarrow 1$ & $1.371(09)$ & $5.494(09)$ \\
\hline 16 & $2 \mathrm{p}_{1 / 2}^{1} 2 \mathrm{p}_{3 / 2}^{3}(1.0) 3 \mathrm{p}_{1 / 2}^{1}$ & ${ }^{4} \mathrm{D}_{1 / 2}$ & 6541286. & $\rightarrow 7$ & 2.127(09) & $\rightarrow 6$ & $9.604(08)$ & $3.675(09)$ \\
\hline 17 & $2 \mathrm{p}_{1 / 2}^{1} 2 \mathrm{p}_{3 / 2}^{3}(1.0) 3 \mathrm{p}_{1 / 2}^{1}$ & ${ }^{4} \mathrm{D}_{3 / 2}^{(20)}$ & 6543742. & $\rightarrow 5$ & $4.154(09)$ & $\rightarrow 6$ & $6.608(08)$ & $6.694(09)$ \\
\hline 18 & $2 \mathrm{p}_{3 / 2}^{4} 3 \mathrm{~s}_{1 / 2}^{1}$ & ${ }^{2} \mathrm{~S}_{1 / 2}$ & 6543747. & $\rightarrow 2$ & $8.909(11)$ & $\rightarrow 1$ & $2.285(11)$ & $1.119(12)$ \\
\hline 19 & $2 \mathrm{p}_{1 / 2}^{2} 2 \mathrm{p}_{3 / 2}^{2}(0.0) 3 \mathrm{p}_{1 / 2}^{1}$ & ${ }^{4} \mathrm{P}_{1 / 2}^{(13)}$ & 6544845 & $\rightarrow 3$ & $4.541(09)$ & $\rightarrow 6$ & $2.659(09)$ & $8.756(09)$ \\
\hline 20 & $2 \mathrm{p}_{1 / 2}^{2} 2 \mathrm{p}_{3 / 2}^{2}(0.0) 3 \mathrm{p}_{3 / 2}^{1}$ & ${ }^{4} \mathrm{D}_{3 / 2}^{(17)}$ & 6560202. & $\rightarrow 6$ & $1.848(09)$ & $\rightarrow 7$ & $1.134(09)$ & $6.040(09)$ \\
\hline 21 & $2 \mathrm{p}_{1 / 2}^{1} 2 \mathrm{p}_{3 / 2}^{3}(1.0) 3 \mathrm{p}_{3 / 2}^{1}$ & ${ }^{4} \mathrm{D}_{5 / 2}$ & 6576158. & $\rightarrow 7$ & $4.778(09)$ & $\rightarrow 1$ & $4.742(08)$ & $5.607(09)$ \\
\hline 22 & $2 \mathrm{p}_{1 / 2}^{2} 2 \mathrm{p}_{3 / 2}^{2}(0.0) 3 \mathrm{p}_{3 / 2}^{1}$ & ${ }^{4} \mathrm{~S}_{3 / 2}$ & 6579629. & $\rightarrow 3$ & $8.591(09)$ & $\rightarrow 6$ & $2.554(09)$ & $1.389(10)$ \\
\hline 23 & $2 p_{1 / 2}^{1} 2 p_{3 / 2}^{3}(1.0) 3 p_{3 / 2}^{1}$ & ${ }^{2} \mathrm{~S}_{1 / 2}$ & 6595860. & $\rightarrow 8$ & $3.152(09)$ & $\rightarrow 3$ & $5.122(08)$ & $5.417(09)$ \\
\hline 24 & $2 \mathrm{p}_{1 / 2}^{1} 2 \mathrm{p}_{3 / 2}^{3}(1.0) 3 \mathrm{p}_{3 / 2}^{1}$ & ${ }^{2} \mathrm{D}_{3 / 2}$ & 6601705. & $\rightarrow 8$ & $3.421(09)$ & $\rightarrow 3$ & $1.524(09)$ & $8.589(09)$ \\
\hline 25 & $2 \mathrm{p}_{1 / 2}^{1} 2 \mathrm{p}_{3 / 2}^{3}(2.0) 3 \mathrm{p}_{1 / 2}^{1}$ & ${ }^{2} \mathrm{~F}_{5 / 2}$ & 6636343. & $\rightarrow 10$ & $2.558(09)$ & $\rightarrow 9$ & $1.027(09)$ & $4.585(09)$ \\
\hline 26 & $2 \mathrm{p}_{1 / 2}^{1} 2 \mathrm{p}_{3 / 2}^{3}(2.0) 3 \mathrm{p}_{3 / 2}^{1}$ & ${ }^{2} \mathrm{~F}_{7 / 2}$ & 6657675. & $\rightarrow 9$ & $4.805(09)$ & $\rightarrow 1$ & $1.026(09)$ & $5.846(09)$ \\
\hline 27 & $2 \mathrm{p}_{1 / 2}^{1} 2 \mathrm{p}_{3 / 2}^{3}(2.0) 3 \mathrm{p}_{3 / 2}^{1}$ & ${ }^{2} \mathrm{D}_{3 / 2}$ & 6673560. & $\rightarrow 10$ & $5.096(09)$ & $\rightarrow 3$ & $4.278(09)$ & $1.078(10)$ \\
\hline 28 & $2 \mathrm{p}_{1 / 2}^{1} 2 \mathrm{p}_{3 / 2}^{3}(2.0) 3 \mathrm{p}_{3 / 2}^{1}$ & ${ }^{2} \mathrm{D}_{5 / 2}$ & 6685704. & $\rightarrow 9$ & $4.754(09)$ & $\rightarrow 10$ & $1.782(09)$ & $7.406(09)$ \\
\hline 29 & $2 \mathrm{p}_{1 / 2}^{1} 2 \mathrm{p}_{3 / 2}^{3}(2.0) 3 \mathrm{p}_{1 / 2}^{1}$ & ${ }^{2} \mathrm{P}_{3 / 2}$ & 6742966. & $\rightarrow 3$ & $3.681(10)$ & $\rightarrow 5$ & $6.360(09)$ & $5.715(10)$ \\
\hline 30 & $2 \mathrm{p}_{1 / 2}^{1} 2 \mathrm{p}_{3 / 2}^{3}(2.0) 3 \mathrm{p}_{3 / 2}^{1}$ & ${ }^{2} \mathrm{P}_{1 / 2}$ & 6758110. & $\rightarrow 3$ & $6.865(10)$ & $\rightarrow 10$ & 7.389(09) & $8.117(10)$ \\
\hline 31 & $2 \mathrm{p}_{1 / 2}^{2} 2 \mathrm{p}_{3 / 2}^{2}(2.0) 3 \mathrm{~d}_{3 / 2}^{1}$ & ${ }^{4} \mathrm{D}_{5 / 2}$ & 6792559. & $\rightarrow 12$ & $4.410(09)$ & $\rightarrow 11$ & $2.911(09)$ & $8.346(09)$ \\
\hline 32 & $2 \mathrm{p}_{1 / 2}^{2} 2 \mathrm{p}_{3 / 2}^{2}(2.0) 3 \mathrm{~d}_{5 / 2}^{1}$ & ${ }^{4} \mathrm{D}_{7 / 2}$ & 6792763. & $\rightarrow 12$ & $4.683(09)$ & $\rightarrow 14$ & $1.874(09)$ & 7.195(09) \\
\hline 33 & $2 \mathrm{p}_{1 / 2}^{2} 2 \mathrm{p}_{3 / 2}^{2}(2.0) 3 \mathrm{~d}_{3 / 2}^{1}$ & ${ }^{4} \mathrm{D}_{3 / 2}^{(46)}$ & 6798295. & $\rightarrow 2$ & $4.236(10)$ & $\rightarrow 11$ & $5.512(09)$ & $5.343(10)$ \\
\hline 34 & $2 \mathrm{p}_{1 / 2}^{2} 2 \mathrm{p}_{3 / 2}^{2}(2.0) 3 \mathrm{~d}_{3 / 2}^{1}$ & ${ }^{4} \mathrm{D}_{1 / 2}^{(42)}$ & 6807665 & $\rightarrow 2$ & $4.395(10)$ & $\rightarrow 11$ & $5.117(09)$ & $5.362(10)$ \\
\hline 35 & $2 \mathrm{p}_{1 / 2}^{2} 2 \mathrm{p}_{3 / 2}^{2}(2.0) 3 \mathrm{~d}_{5 / 2}^{1}$ & ${ }^{4} \mathrm{~F}_{9 / 2}$ & 6817655. & $\rightarrow 14$ & 7.978(09) & $\rightarrow 4$ & $2.692(05)$ & $7.979(09)$ \\
\hline 36 & $2 \mathrm{p}_{3 / 2}^{4} 3 \mathrm{p}_{3 / 2}^{1}$ & ${ }^{2} \mathrm{P}_{3 / 2}$ & 6824037. & $\rightarrow 3$ & $1.928(10)$ & $\rightarrow 18$ & $5.300(09)$ & $2.592(10)$ \\
\hline 37 & $2 \mathrm{p}_{1 / 2}^{2} 2 \mathrm{p}_{3 / 2}^{2}(2.0) 3 \mathrm{~d}_{3 / 2}^{1}$ & ${ }^{2} \mathrm{~F}_{7 / 2}$ & 6828878. & $\rightarrow 15$ & $7.758(09)$ & $\rightarrow 12$ & $1.070(09)$ & $9.028(09)$ \\
\hline 38 & $2 \mathrm{p}_{1 / 2}^{2} 2 \mathrm{p}_{3 / 2}^{2}(2.0) 3 \mathrm{~d}_{5 / 2}^{1}$ & ${ }^{4} \mathrm{P}_{1 / 2}$ & 6845037. & $\rightarrow 1$ & $2.637(12)$ & $\rightarrow 11$ & $3.845(09)$ & $2.646(12)$ \\
\hline 39 & $2 \mathrm{p}_{3 / 2}^{4} 3 \mathrm{p}_{1 / 2}^{1}$ & ${ }^{2} \mathrm{P}_{1 / 2}$ & 6848296. & $\rightarrow 18$ & $5.228(09)$ & $\rightarrow 8$ & 4.683(09) & $1.762(10)$ \\
\hline 40 & $2 \mathrm{p}_{1 / 2}^{2} 2 \mathrm{p}_{3 / 2}^{2}(2.0) 3 \mathrm{~d}_{5 / 2}^{1}$ & ${ }^{4} \mathrm{P}_{3 / 2}$ & 6860872. & $\rightarrow 1$ & $3.265(12)$ & $\rightarrow 2$ & $4.396(11)$ & $3.713(12)$ \\
\hline 41 & $2 \mathrm{p}_{1 / 2}^{2} 2 \mathrm{p}_{3 / 2}^{2}(2.0) 3 \mathrm{~d}_{5 / 2}^{1}$ & ${ }^{2} \mathrm{~F}_{5 / 2}$ & 6869054. & $\rightarrow 1$ & $4.310(12)$ & $\rightarrow 17$ & 4.297(09) & $4.319(12)$ \\
\hline 42 & $2 \mathrm{p}_{1 / 2}^{1} 2 \mathrm{p}_{3 / 2}^{3}(1.0) 3 \mathrm{~d}_{3 / 2}^{1}$ & ${ }^{4} \mathrm{D}_{1 / 2}^{(34)}$ & 6886694. & $\rightarrow 1$ & $1.857(11)$ & $\rightarrow 2$ & $1.112(11)$ & $3.052(11)$ \\
\hline
\end{tabular}

found, while differences with the Cornille et al. (1992) data are $16 \%$. Our probabilities for most transitions are slightly smaller than the corresponding experimental values.
The greatest deviation from experiment is for the E1 transition from level $61\left(2 \mathrm{p}_{3 / 2}^{4} 3 \mathrm{~d}_{3 / 2}^{1}{ }^{2} \mathrm{D}_{3 / 2}\right)$ to the ground state. Our transition probability is $35 \%$ smaller than that by NIST, while 
Table 1. continued.

\begin{tabular}{|c|c|c|c|c|c|c|c|c|}
\hline Index & $\begin{array}{l}\text { Configuration } \\
\text { (jj scheme) }\end{array}$ & LSJ & $E\left(\mathrm{~cm}^{-1}\right)$ & $\begin{array}{l}\text { final } \\
\text { level }\end{array}$ & $A^{r}\left(\mathrm{~s}^{-1}\right)$ & $\begin{array}{l}\text { final } \\
\text { level }\end{array}$ & $A^{r}\left(\mathrm{~s}^{-1}\right)$ & $\sum A^{r}\left(\mathrm{~s}^{-1}\right)$ \\
\hline 43 & $2 \mathrm{p}_{1 / 2}^{2} 2 \mathrm{p}_{3 / 2}^{2}(0.0) 3 \mathrm{~d}_{3 / 2}^{1}$ & ${ }^{4} \mathrm{~F}_{3 / 2}$ & 6891781. & $\rightarrow 1$ & $6.758(11)$ & $\rightarrow 2$ & $7.847(10)$ & $7.636(11)$ \\
\hline 44 & $2 \mathrm{p}_{1 / 2}^{2} 2 \mathrm{p}_{3 / 2}^{2}(0.0) 3 \mathrm{~d}_{5 / 2}^{1}$ & ${ }^{4} \mathrm{~F}_{5 / 2}$ & 6892755. & $\rightarrow 20$ & 4.552(09) & $\rightarrow 17$ & $1.523(09)$ & $8.816(09)$ \\
\hline 45 & $2 \mathrm{p}_{1 / 2}^{1} 2 \mathrm{p}_{3 / 2}^{3}(1.0) 3 \mathrm{~d}_{5 / 2}^{1}$ & ${ }^{4} \mathrm{~F}_{7 / 2}$ & 6902043. & $\rightarrow 21$ & $7.625(09)$ & $\rightarrow 14$ & $1.761(08)$ & 7.821(09) \\
\hline 46 & $2 \mathrm{p}_{1 / 2}^{1} 2 \mathrm{p}_{3 / 2}^{3}(1.0) 3 \mathrm{~d}_{3 / 2}^{1}$ & ${ }^{4} \mathrm{D}_{3 / 2}^{(33)}$ & 6906682. & $\rightarrow 1$ & $9.267(11)$ & $\rightarrow 2$ & $7.654(11)$ & $1.701(12)$ \\
\hline 47 & $2 \mathrm{p}_{1 / 2}^{1} 2 \mathrm{p}_{3 / 2}^{3}(1.0) 3 \mathrm{~d}_{5 / 2}^{1}$ & ${ }^{4} \mathrm{P}_{5 / 2}$ & 6922310. & $\rightarrow 1$ & $4.001(11)$ & $\rightarrow 24$ & $3.727(09)$ & $4.087(11)$ \\
\hline 48 & $2 \mathrm{p}_{1 / 2}^{1} 2 \mathrm{p}_{3 / 2}^{3}(1.0) 3 \mathrm{~d}_{5 / 2}^{1}$ & ${ }^{2} \mathrm{P}_{3 / 2}$ & 6935515. & $\rightarrow 2$ & $1.051(12)$ & $\rightarrow 1$ & $2.288(11)$ & $1.288(12)$ \\
\hline 49 & $2 \mathrm{p}_{1 / 2}^{1} 2 \mathrm{p}_{3 / 2}^{3}(1.0) 3 \mathrm{~d}_{3 / 2}^{1}$ & ${ }^{2} \mathrm{D}_{5 / 2}$ & 6947225. & $\rightarrow 1$ & $6.908(12)$ & $\rightarrow 24$ & $3.443(09)$ & $6.917(12)$ \\
\hline 50 & $2 \mathrm{p}_{1 / 2}^{1} 2 \mathrm{p}_{3 / 2}^{3}(2.0) 3 \mathrm{~d}_{3 / 2}^{1}$ & ${ }^{2} \mathrm{G}_{7 / 2}$ & 6977270. & $\rightarrow 25$ & 7.919(09) & $\rightarrow 26$ & $5.246(08)$ & 8.611(09) \\
\hline 51 & $2 \mathrm{p}_{1 / 2}^{1} 2 \mathrm{p}_{3 / 2}^{3}(2.0) 3 \mathrm{~d}_{5 / 2}^{1}$ & ${ }^{2} \mathrm{G}_{9 / 2}$ & 6979625. & $\rightarrow 26$ & 7.421(09) & $\rightarrow 14$ & $8.741(06)$ & 7.430(09) \\
\hline 52 & $2 \mathrm{p}_{1 / 2}^{1} 2 \mathrm{p}_{3 / 2}^{3}(2.0) 3 \mathrm{~d}_{5 / 2}^{1}$ & ${ }^{2} \mathrm{~F}_{5 / 2}$ & 7005068. & $\rightarrow 1$ & $1.363(12)$ & $\rightarrow 27$ & $5.706(09)$ & $1.372(12)$ \\
\hline 53 & $2 \mathrm{p}_{1 / 2}^{1} 2 \mathrm{p}_{3 / 2}^{3}(2.0) 3 \mathrm{~d}_{3 / 2}^{1}$ & ${ }^{2} S_{1 / 2}$ & 7005754 & $\rightarrow 1$ & $1.474(13)$ & $\rightarrow 2$ & $2.812(12)$ & $1.756(13)$ \\
\hline 54 & $2 \mathrm{p}_{1 / 2}^{1} 2 \mathrm{p}_{3 / 2}^{3}(2.0) 3 \mathrm{~d}_{5 / 2}^{1}$ & ${ }^{2} \mathrm{~F}_{7 / 2}$ & 7015684 & $\rightarrow 28$ & $5.913(09)$ & $\rightarrow 26$ & $3.083(09)$ & $9.110(09)$ \\
\hline 55 & $2 \mathrm{p}_{1 / 2}^{1} 2 \mathrm{p}_{3 / 2}^{3}(2.0) 3 \mathrm{~d}_{3 / 2}^{1}$ & ${ }^{2} \mathrm{P}_{3 / 2}$ & 7033450 . & $\rightarrow 1$ & $1.929(13)$ & $\rightarrow 2$ & $2.839(12)$ & $2.214(13)$ \\
\hline 56 & $2 \mathrm{p}_{1 / 2}^{1} 2 \mathrm{p}_{3 / 2}^{3}(2.0) 3 \mathrm{~d}_{3 / 2}^{1}$ & ${ }^{2} \mathrm{D}_{5 / 2}$ & 7035986. & $\rightarrow 1$ & $1.991(13)$ & $\rightarrow 25$ & $3.331(09)$ & $1.992(13)$ \\
\hline 57 & $2 \mathrm{p}_{1 / 2}^{1} 2 \mathrm{p}_{3 / 2}^{3}(2.0) 3 \mathrm{~d}_{3 / 2}^{1}$ & ${ }^{2} \mathrm{D}_{3 / 2}$ & 7060411. & $\rightarrow 2$ & $1.333(13)$ & $\rightarrow 1$ & $3.944(12)$ & $1.728(13)$ \\
\hline 58 & $2 \mathrm{p}_{1 / 2}^{1} 2 \mathrm{p}_{3 / 2}^{3}(2.0) 3 \mathrm{~d}_{5 / 2}^{1}$ & ${ }^{2} \mathrm{P}_{1 / 2}$ & 7070305 . & $\rightarrow 2$ & $2.133(13)$ & $\rightarrow 1$ & $4.633(12)$ & $2.598(13)$ \\
\hline 59 & $2 \mathrm{p}_{3 / 2}^{4} 3 \mathrm{~d}_{5 / 2}^{1}$ & ${ }^{2} \mathrm{D}_{5 / 2}$ & 7153608. & $\rightarrow 1$ & $1.152(12)$ & $\rightarrow 36$ & $8.013(09)$ & $1.161(12)$ \\
\hline 60 & $2 \mathrm{~s}_{1 / 2}^{1} 2 \mathrm{p}_{1 / 2}^{2} 2 \mathrm{p}_{3 / 2}^{3}(2.0) 3 \mathrm{~s}_{1 / 2}^{1}$ & ${ }^{4} \mathrm{P}_{5 / 2}$ & 7157893. & $\rightarrow 4$ & $4.461(10)$ & $\rightarrow 7$ & $1.034(10)$ & $6.366(10)$ \\
\hline 61 & $2 \mathrm{p}_{3 / 2}^{4} 3 \mathrm{~d}_{3 / 2}^{1}$ & ${ }^{2} \mathrm{D}_{3 / 2}$ & 7175002 . & $\rightarrow 2$ & $1.436(13)$ & $\rightarrow 1$ & $6.240(10)$ & $1.444(13)$ \\
\hline 62 & $2 \mathrm{~s}_{1 / 2}^{1} 2 \mathrm{p}_{1 / 2}^{2} 2 \mathrm{p}_{3 / 2}^{3}$ (2.0) $3 \mathrm{~s}_{1 / 2}^{1}$ & ${ }^{4} \mathrm{P}_{3 / 2}$ & 7194980. & $\rightarrow 3$ & $4.542(11)$ & $\rightarrow 4$ & $2.789(10)$ & $5.178(11)$ \\
\hline 63 & $2 \mathrm{~s}_{1 / 2}^{1} 2 \mathrm{p}_{1 / 2}^{2} 2 \mathrm{p}_{3 / 2}^{3}(1.0) 3 \mathrm{~s}_{1 / 2}^{1}$ & ${ }^{4} \mathrm{P}_{1 / 2}$ & 7240155. & $\rightarrow 3$ & $2.898(11)$ & $\rightarrow 5$ & $3.832(10)$ & $3.630(11)$ \\
\hline 64 & $2 \mathrm{~s}_{1 / 2}^{1} 2 \mathrm{p}_{1 / 2}^{1}(1.0) 2 \mathrm{p}_{3 / 2}^{4} 3 \mathrm{~s}_{1 / 2}^{1}$ & ${ }^{2} \mathrm{P}_{3 / 2}$ & 7251175 . & $\rightarrow 3$ & $8.600(11)$ & $\rightarrow 7$ & $1.631(10)$ & $9.102(11)$ \\
\hline 65 & $2 \mathrm{~s}_{1 / 2}^{1} 2 \mathrm{p}_{1 / 2}^{1}(0.0) 2 \mathrm{p}_{3 / 2}^{4} 3 \mathrm{~s}_{1 / 2}^{1}$ & ${ }^{2} \mathrm{P}_{1 / 2}$ & 7304841. & $\rightarrow 3$ & $1.506(12)$ & $\rightarrow 8$ & $2.896(10)$ & $1.569(12)$ \\
\hline 66 & $2 \mathrm{~s}_{1 / 2}^{1} 2 \mathrm{p}_{1 / 2}^{2} 2 \mathrm{p}_{3 / 2}^{3}(2.0) 3 \mathrm{p}_{1 / 2}^{1}$ & ${ }^{4} S_{3 / 2}$ & 7391763. & $\rightarrow 11$ & $2.727(10)$ & $\rightarrow 12$ & $1.743(10)$ & $7.188(10)$ \\
\hline 67 & $2 \mathrm{~s}_{1 / 2}^{1} 2 \mathrm{p}_{1 / 2}^{2} 2 \mathrm{p}_{3 / 2}^{3}(2.0) 3 \mathrm{p}_{1 / 2}^{1}$ & ${ }^{4} \mathrm{D}_{5 / 2}^{(74)}$ & 7421035 . & $\rightarrow 1$ & $7.225(11)$ & $\rightarrow 12$ & $2.542(10)$ & $7.806(11)$ \\
\hline 68 & $2 \mathrm{~s}_{1 / 2}^{1} 2 \mathrm{p}_{1 / 2}^{2} 2 \mathrm{p}_{3 / 2}^{3}(2.0) 3 \mathrm{p}_{3 / 2}^{1}$ & ${ }^{4} \mathrm{D}_{7 / 2}$ & 7429666. & $\rightarrow 14$ & $3.447(10)$ & $\rightarrow 21$ & $6.818(09)$ & $5.494(10)$ \\
\hline 69 & $2 \mathrm{~s}_{1 / 2}^{1} 2 \mathrm{p}_{1 / 2}^{2} 2 \mathrm{p}_{3 / 2}^{3}$ (2.0) $3 \mathrm{p}_{3 / 2}^{1}$ & ${ }^{4} \mathrm{D}_{3 / 2}$ & 7446302. & $\rightarrow 1$ & $1.178(12)$ & $\rightarrow 17$ & $1.434(10)$ & $1.247(12)$ \\
\hline 70 & $2 \mathrm{~s}_{1 / 2}^{1} 2 \mathrm{p}_{1 / 2}^{2} 2 \mathrm{p}_{3 / 2}^{3}(2.0) 3 \mathrm{p}_{3 / 2}^{1}$ & ${ }^{2} \mathrm{D}_{5 / 2}$ & 7459550. & $\rightarrow 1$ & $1.673(12)$ & $\rightarrow 15$ & $1.450(10)$ & $1.737(12)$ \\
\hline 71 & $2 \mathrm{~s}_{1 / 2}^{1} 2 \mathrm{p}_{1 / 2}^{2} 2 \mathrm{p}_{3 / 2}^{3}$ (2.0) $3 \mathrm{p}_{3 / 2}^{1}$ & ${ }^{4} \mathrm{D}_{1 / 2}$ & 7473083. & $\rightarrow 1$ & $2.694(11)$ & $\rightarrow 2$ & $1.980(11)$ & $5.312(11)$ \\
\hline 72 & $2 \mathrm{~s}_{1 / 2}^{1} 2 \mathrm{p}_{1 / 2}^{1}(1.0) 2 \mathrm{p}_{3 / 2}^{4} 3 \mathrm{p}_{1 / 2}^{1}$ & ${ }^{2} \mathrm{P}_{3 / 2}$ & 7486040 . & $\rightarrow 1$ & $2.394(12)$ & $\rightarrow 2$ & $2.847(11)$ & $2.745(12)$ \\
\hline 73 & $2 \mathrm{~s}_{1 / 2}^{1} 2 \mathrm{p}_{1 / 2}^{2} 2 \mathrm{p}_{3 / 2}^{3}$ (2.0) $3 \mathrm{p}_{3 / 2}^{1}$ & ${ }^{2} \mathrm{P}_{1 / 2}$ & 7504936. & $\rightarrow 1$ & $3.476(12)$ & $\rightarrow 2$ & $3.159(11)$ & $3.869(12)$ \\
\hline 74 & $2 \mathrm{~s}_{1 / 2}^{1} 2 \mathrm{p}_{1 / 2}^{2} 2 \mathrm{p}_{3 / 2}^{3}$ (1.0) $3 \mathrm{p}_{3 / 2}^{1}$ & ${ }^{4} \mathrm{D}_{5 / 2}^{(67)}$ & 7505906. & $\rightarrow 1$ & $1.182(12)$ & $\rightarrow 14$ & $2.960(10)$ & $1.252(12)$ \\
\hline 75 & $2 \mathrm{~s}_{1 / 2}^{1} 2 \mathrm{p}_{1 / 2}^{1}(1.0) 2 \mathrm{p}_{3 / 2}^{4} 3 \mathrm{p}_{3 / 2}^{1}$ & ${ }^{4} \mathrm{P}_{3 / 2}$ & 7510542 . & $\rightarrow 2$ & $7.037(11)$ & $\rightarrow 1$ & $4.847(11)$ & $1.261(12)$ \\
\hline 76 & $2 \mathrm{~s}_{1 / 2}^{1} 2 \mathrm{p}_{1 / 2}^{1}(0.0) 2 \mathrm{p}_{3 / 2}^{4} 3 \mathrm{p}_{1 / 2}^{1}$ & ${ }^{4} \mathrm{P}_{1 / 2}$ & 7515405. & $\rightarrow 2$ & $4.081(10)$ & $\rightarrow 17$ & $3.922(10)$ & $1.423(11)$ \\
\hline 77 & $2 \mathrm{~s}_{1 / 2}^{1} 2 \mathrm{p}_{1 / 2}^{2} 2 \mathrm{p}_{3 / 2}^{3}(1.0) 3 \mathrm{~s}_{1 / 2}^{1}$ & ${ }^{2} \mathrm{P}_{3 / 2}$ & 7526394. & $\rightarrow 3$ & $8.829(11)$ & $\rightarrow 9$ & $1.481(11)$ & $1.074(12)$ \\
\hline 78 & $2 \mathrm{~s}_{1 / 2}^{1} 2 \mathrm{p}_{1 / 2}^{1}(1.0) 2 \mathrm{p}_{3 / 2}^{4} 3 \mathrm{~s}_{1 / 2}^{1}$ & ${ }^{2} \mathrm{P}_{1 / 2}$ & 7533542. & $\rightarrow 3$ & $3.505(11)$ & $\rightarrow 10$ & $1.464(11)$ & $5.285(11)$ \\
\hline 79 & $2 \mathrm{~s}_{1 / 2}^{1} 2 \mathrm{p}_{1 / 2}^{1}(0.0) 2 \mathrm{p}_{3 / 2}^{4} 3 \mathrm{p}_{3 / 2}^{1}$ & ${ }^{2} \mathrm{D}_{3 / 2}$ & 7555364. & $\rightarrow 2$ & $1.743(12)$ & $\rightarrow 21$ & $2.966(10)$ & $1.822(12)$ \\
\hline 80 & $2 \mathrm{~s}_{1 / 2}^{1} 2 \mathrm{p}_{1 / 2}^{1}(1.0) 2 \mathrm{p}_{3 / 2}^{4} 3 \mathrm{p}_{3 / 2}^{1}$ & ${ }^{2} \mathrm{~S}_{1 / 2}$ & 7582841. & $\rightarrow 2$ & $3.320(12)$ & $\rightarrow 1$ & $1.135(12)$ & $4.543(12)$ \\
\hline 81 & $2 \mathrm{~s}_{1 / 2}^{1} 2 \mathrm{p}_{1 / 2}^{2} 2 \mathrm{p}_{3 / 2}^{3}(2.0) 3 \mathrm{~d}_{3 / 2}^{1}$ & ${ }^{4} \mathrm{P}_{1 / 2}$ & 7719622. & $\rightarrow 33$ & $2.540(10)$ & $\rightarrow 34$ & $1.773(10)$ & 7.091(10) \\
\hline 82 & $2 \mathrm{~s}_{1 / 2}^{1} 2 \mathrm{p}_{1 / 2}^{2} 2 \mathrm{p}_{3 / 2}^{3}$ (2.0) $3 \mathrm{~d}_{3 / 2}^{1}$ & ${ }^{4} \mathrm{P}_{3 / 2}$ & 7728609. & $\rightarrow 3$ & $2.827(10)$ & $\rightarrow 31$ & $2.703(10)$ & $8.940(10)$ \\
\hline 83 & $2 \mathrm{~s}_{1 / 2}^{1} 2 \mathrm{p}_{1 / 2}^{2} 2 \mathrm{p}_{3 / 2}^{3}(2.0) 3 \mathrm{~d}_{5 / 2}^{1}$ & ${ }^{4} \mathrm{~F}_{9 / 2}$ & 7737951. & $\rightarrow 35$ & $2.559(10)$ & $\rightarrow 68$ & 7.001(09) & $4.226(10)$ \\
\hline 84 & $2 \mathrm{~s}_{1 / 2}^{1} 2 \mathrm{p}_{1 / 2}^{2} 2 \mathrm{p}_{3 / 2}^{3}(2.0) 3 \mathrm{~d}_{5 / 2}^{1}$ & ${ }^{4} \mathrm{P}_{5 / 2}$ & 7744392. & $\rightarrow 32$ & $2.471(10)$ & $\rightarrow 31$ & $9.873(09)$ & $5.908(10)$ \\
\hline 85 & $2 \mathrm{~s}_{1 / 2}^{1} 2 \mathrm{p}_{1 / 2}^{2} 2 \mathrm{p}_{3 / 2}^{3}(2.0) 3 \mathrm{~d}_{3 / 2}^{1}$ & ${ }^{4} \mathrm{~F}_{7 / 2}$ & 7749362. & $\rightarrow 37$ & $1.185(10)$ & $\rightarrow 32$ & $1.103(10)$ & $4.550(10)$ \\
\hline 86 & $2 \mathrm{~s}_{1 / 2}^{1} 2 \mathrm{p}_{1 / 2}^{2} 2 \mathrm{p}_{3 / 2}^{3}(2.0) 3 \mathrm{~d}_{3 / 2}^{1}$ & ${ }^{4} \mathrm{~F}_{5 / 2}$ & 7769828. & $\rightarrow 32$ & $9.247(09)$ & $\rightarrow 44$ & $9.130(09)$ & $5.127(10)$ \\
\hline 87 & $2 \mathrm{~s}_{1 / 2}^{1} 2 \mathrm{p}_{1 / 2}^{1}(1.0) 2 \mathrm{p}_{3 / 2}^{4} 3 \mathrm{p}_{1 / 2}^{1}$ & ${ }^{2} \mathrm{D}_{3 / 2}$ & 7770006. & $\rightarrow 2$ & $1.231(12)$ & $\rightarrow 1$ & $3.157(11)$ & $1.742(12)$ \\
\hline 88 & $2 \mathrm{~s}_{1 / 2}^{1} 2 \mathrm{p}_{1 / 2}^{2} 2 \mathrm{p}_{3 / 2}^{3}(2.0) 3 \mathrm{~d}_{5 / 2}^{1}$ & ${ }^{2} \mathrm{~F}_{7 / 2}^{(95)}$ & 7784003. & $\rightarrow 35$ & $1.456(10)$ & $\rightarrow 37$ & $9.164(09)$ & $5.481(10)$ \\
\hline 89 & $2 \mathrm{~s}_{1 / 2}^{1} 2 \mathrm{p}_{1 / 2}^{2} 2 \mathrm{p}_{3 / 2}^{3}(2.0) 3 \mathrm{~d}_{5 / 2}^{1}$ & ${ }^{4} \mathrm{~F}_{3 / 2}$ & 7787953. & $\rightarrow 3$ & $5.289(11)$ & $\rightarrow 43$ & $1.662(10)$ & $5.807(11)$ \\
\hline 90 & $2 \mathrm{~s}_{1 / 2}^{1} 2 \mathrm{p}_{1 / 2}^{1}(1.0) 2 \mathrm{p}_{3 / 2}^{4} 3 \mathrm{p}_{3 / 2}^{1}$ & ${ }^{2} \mathrm{D}_{5 / 2}$ & 7792996. & $\rightarrow 1$ & $9.156(11)$ & $\rightarrow 26$ & $1.378(11)$ & $1.112(12)$ \\
\hline 91 & $2 \mathrm{~s}_{1 / 2}^{1} 2 \mathrm{p}_{1 / 2}^{2} 2 \mathrm{p}_{3 / 2}^{3}(1.0) 3 \mathrm{p}_{1 / 2}^{1}$ & ${ }^{2} \mathrm{P}_{1 / 2}$ & 7802019. & $\rightarrow 2$ & $1.284(12)$ & $\rightarrow 1$ & $1.600(11)$ & $1.628(12)$ \\
\hline
\end{tabular}


Table 1. continued.

\begin{tabular}{|c|c|c|c|c|c|c|c|c|}
\hline Index & $\begin{array}{l}\text { Configuration } \\
\text { (jj scheme) }\end{array}$ & LSJ & $E\left(\mathrm{~cm}^{-1}\right)$ & $\begin{array}{l}\text { final } \\
\text { level }\end{array}$ & $A^{r}\left(\mathrm{~s}^{-1}\right)$ & $\begin{array}{l}\text { final } \\
\text { level }\end{array}$ & $A^{r}\left(\mathrm{~s}^{-1}\right)$ & $\sum A^{r}\left(\mathrm{~s}^{-1}\right)$ \\
\hline 92 & $2 \mathrm{~s}_{1 / 2}^{1} 2 \mathrm{p}_{1 / 2}^{2} 2 \mathrm{p}_{3 / 2}^{3}(1.0) 3 \mathrm{p}_{3 / 2}^{1}$ & ${ }^{2} \mathrm{P}_{3 / 2}$ & 7812627. & $\rightarrow 2$ & $6.243(11)$ & $\rightarrow 1$ & $4.263(11)$ & $1.237(12)$ \\
\hline 93 & $2 \mathrm{~s}_{1 / 2}^{1} 2 \mathrm{p}_{1 / 2}^{1}(1.0) 2 \mathrm{p}_{3 / 2}^{4} 3 \mathrm{~d}_{3 / 2}^{1}$ & ${ }^{4} \mathrm{D}_{1 / 2}$ & 7817786. & $\rightarrow 3$ & $1.918(12)$ & $\rightarrow 43$ & $2.683(10)$ & $1.976(12)$ \\
\hline 94 & $2 \mathrm{~s}_{1 / 2}^{1} 2 \mathrm{p}_{1 / 2}^{1}(1.0) 2 \mathrm{p}_{3 / 2}^{4} 3 \mathrm{~d}_{5 / 2}^{1}$ & ${ }^{2} \mathrm{~F}_{5 / 2}^{(101)}$ & 7828023. & $\rightarrow 44$ & $1.034(10)$ & $\rightarrow 45$ & $9.362(09)$ & $6.154(10)$ \\
\hline 95 & $2 \mathrm{~s}_{1 / 2}^{1} 2 \mathrm{p}_{1 / 2}^{1}(1.0) 2 \mathrm{p}_{3 / 2}^{4} 3 \mathrm{~d}_{5 / 2}^{1}$ & ${ }^{2} \mathrm{~F}_{7 / 2}^{(88)}$ & 7829672. & $\rightarrow 35$ & $2.293(10)$ & $\rightarrow 45$ & $8.857(09)$ & $6.160(10)$ \\
\hline 96 & $2 \mathrm{~s}_{1 / 2}^{1} 2 \mathrm{p}_{1 / 2}^{2} 2 \mathrm{p}_{3 / 2}^{3}(1.0) 3 \mathrm{~d}_{3 / 2}^{1}$ & ${ }^{4} \mathrm{D}_{5 / 2}$ & 7830215 . & $\rightarrow 37$ & $3.509(10)$ & $\rightarrow 72$ & $5.749(09)$ & $6.377(10)$ \\
\hline 97 & $2 \mathrm{~s}_{1 / 2}^{1} 2 \mathrm{p}_{1 / 2}^{2} 2 \mathrm{p}_{3 / 2}^{3}(1.0) 3 \mathrm{~d}_{5 / 2}^{1}$ & ${ }^{4} \mathrm{D}_{3 / 2}$ & 7838102. & $\rightarrow 3$ & $1.314(11)$ & $\rightarrow 44$ & $1.782(10)$ & $1.913(11)$ \\
\hline 98 & $2 \mathrm{~s}_{1 / 2}^{1} 2 \mathrm{p}_{1 / 2}^{1}(0.0) 2 \mathrm{p}_{3 / 2}^{4} 3 \mathrm{~d}_{3 / 2}^{1}$ & ${ }^{2} \mathrm{D}_{3 / 2}$ & 7843566. & $\rightarrow 3$ & $2.512(12)$ & $\rightarrow 41$ & $1.866(10)$ & $2.579(12)$ \\
\hline 99 & $2 \mathrm{~s}_{1 / 2}^{1} 2 \mathrm{p}_{1 / 2}^{2} 2 \mathrm{p}_{3 / 2}^{3}(1.0) 3 \mathrm{p}_{3 / 2}^{1}$ & ${ }^{2} \mathrm{~S}_{1 / 2}$ & 7847512 . & $\rightarrow 29$ & $7.428(10)$ & $\rightarrow 39$ & $1.974(10)$ & $1.563(11)$ \\
\hline 100 & $2 \mathrm{~s}_{1 / 2}^{1} 2 \mathrm{p}_{1 / 2}^{2} 2 \mathrm{p}_{3 / 2}^{3}(2.0) 3 \mathrm{~d}_{5 / 2}^{1}$ & ${ }^{2} \mathrm{P}_{1 / 2}$ & 7871247. & $\rightarrow 3$ & $1.281(13)$ & $\rightarrow 46$ & $2.347(10)$ & $1.291(13)$ \\
\hline 101 & $2 \mathrm{~s}_{1 / 2}^{1} 2 \mathrm{p}_{1 / 2}^{1}(0.0) 2 \mathrm{p}_{3 / 2}^{4} 3 \mathrm{~d}_{5 / 2}^{1}$ & ${ }^{2} \mathrm{~F}_{5 / 2}^{(94)}$ & 7880249. & $\rightarrow 45$ & $2.320(10)$ & $\rightarrow 47$ & $1.111(10)$ & $6.321(10)$ \\
\hline 102 & $2 \mathrm{~s}_{1 / 2}^{1} 2 \mathrm{p}_{1 / 2}^{1}(0.0) 2 \mathrm{p}_{3 / 2}^{4} 3 \mathrm{~d}_{3 / 2}^{1}$ & ${ }^{2} \mathrm{P}_{3 / 2}$ & 7929150. & $\rightarrow 3$ & $1.460(13)$ & $\rightarrow 49$ & $3.970(10)$ & $1.469(13)$ \\
\hline 103 & $2 \mathrm{~s}_{1 / 2}^{1} 2 \mathrm{p}_{1 / 2}^{1}(1.0) 2 \mathrm{p}_{3 / 2}^{4} 3 \mathrm{~d}_{3 / 2}^{1}$ & ${ }^{2} \mathrm{~F}_{5 / 2}$ & 8109407. & $\rightarrow 50$ & $1.267(11)$ & $\rightarrow 37$ & $1.179(10)$ & $1.792(11)$ \\
\hline 104 & $2 \mathrm{~s}_{1 / 2}^{1} 2 \mathrm{p}_{1 / 2}^{2} 2 \mathrm{p}_{3 / 2}^{3}(1.0) 3 \mathrm{~d}_{5 / 2}^{1}$ & ${ }^{2} \mathrm{~F}_{7 / 2}$ & 8110287. & $\rightarrow 51$ & $1.231(11)$ & $\rightarrow 54$ & $2.027(10)$ & $1.769(11)$ \\
\hline 105 & $2 \mathrm{~s}_{1 / 2}^{1} 2 \mathrm{p}_{1 / 2}^{2} 2 \mathrm{p}_{3 / 2}^{3}(1.0) 3 \mathrm{~d}_{5 / 2}^{1}$ & ${ }^{2} \mathrm{P}_{3 / 2}$ & 8120581. & $\rightarrow 3$ & $1.370(13)$ & $\rightarrow 52$ & $2.825(10)$ & $1.382(13)$ \\
\hline 106 & $2 \mathrm{~s}_{1 / 2}^{1} 2 \mathrm{p}_{1 / 2}^{1}(1.0) 2 \mathrm{p}_{3 / 2}^{4} 3 \mathrm{~d}_{3 / 2}^{1}$ & ${ }^{2} \mathrm{P}_{1 / 2}$ & 8129486. & $\rightarrow 3$ & $1.941(13)$ & $\rightarrow 57$ & $2.744(10)$ & $1.953(13)$ \\
\hline 107 & $2 \mathrm{~s}_{1 / 2}^{1} 2 \mathrm{p}_{1 / 2}^{2} 2 \mathrm{p}_{3 / 2}^{3}(1.0) 3 \mathrm{~d}_{5 / 2}^{1}$ & ${ }^{2} \mathrm{D}_{5 / 2}$ & 8136486. & $\rightarrow 54$ & $7.584(10)$ & $\rightarrow 52$ & $2.475(10)$ & $1.518(11)$ \\
\hline 108 & $2 \mathrm{~s}_{1 / 2}^{1} 2 \mathrm{p}_{1 / 2}^{1}(1.0) 2 \mathrm{p}_{3 / 2}^{4} 3 \mathrm{~d}_{3 / 2}^{1}$ & ${ }^{2} \mathrm{D}_{3 / 2}$ & 8137529. & $\rightarrow 3$ & $2.479(12)$ & $\rightarrow 56$ & $4.901(10)$ & $2.628(12)$ \\
\hline
\end{tabular}

the value obtained by Cornille et al. (1992) exceeds the experimental transition probability by $53 \%$. On the other hand, our transition probability from the same level to the first excited level differs from experiment by only $4 \%$.

A comparison of our results and those obtained by Sampson et al. (1991) for transition probabilities in Table 4 shows a similar average deviation. As mentioned above, our largest discrepancy of $35 \%$ from experimental data is for the transition from level $61\left(2 \mathrm{p}_{3 / 2}^{4} 3 \mathrm{~d}_{3 / 2}^{1}{ }^{2} \mathrm{D}_{3 / 2}\right)$ to the ground state, whereas for the Sampson et al. (1991) data the disagreement with experiment is $10 \%$. On the other hand, the transition probability from this level to the ground state is a factor of 1000 smaller than that to first excited level, for which the discrepancies of our and the Sampson et al. results with experiment is similar, while their value is larger than the NIST one and our result is smaller.

The largest deviation between the Coulomb and Babushkin gauges for the transitions presented in Table 4 is obtained for transitions from the $1 s^{2} 2 s^{1} 2 p^{6}{ }^{2} S_{1 / 2}$ level to the fine-structure levels ${ }^{2} \mathrm{P}_{3 / 2}$ and ${ }^{2} \mathrm{P}_{1 / 2}$ of the ground configuration, but this does not exceed $18 \%$. Coulomb and Babushkin gauges in the nonrelativistic limit correspond to the velocity and length forms of the transition operator. The agreement of the results obtained for these two forms may serve as an additional measure for the accuracy of generated atomic state wave functions. The calculated energy of the initial level, i.e. $1 s^{2} 2 s^{1} 2 p^{6}{ }^{2} S_{1 / 2}$, as was mentioned above has also the largest discrepancy with experiment. For other E1 transitions in Table 4 these two forms agree within $10 \%$. The average deviation between length and velocity forms for E1 transitions is 5\%. These two forms coincide for E2 transitions presented in Table 4.

Agreement between the two forms carried out for a large CI basis is never good for weak transitions, and results can differ by over an order of magnitude. Results for these transitions are sensitive to mixing coefficients and as well as
Table 2. Spectroscopic identifications of levels which were changed to ensure the completeness of the spectroscopic dataset. Indexes of levels in the first column are taken from Tables 1 and 5.

\begin{tabular}{|c|c|c|c|}
\hline Index & Initial LSJ & Final LSJ & LS-configuration \\
\hline 19 & ${ }^{4} \mathrm{P}_{1 / 2}$ & ${ }^{2} \mathrm{P}_{1 / 2}$ & $2 s^{2} 2 p^{4}\left({ }^{3} P\right) 3 p^{1}$ \\
\hline 20 & ${ }^{4} \mathrm{D}_{3 / 2}$ & ${ }^{2} \mathrm{P}_{3 / 2}$ & $2 s^{2} 2 p^{4}\left({ }^{3} P\right) 3 p^{1}$ \\
\hline 42 & ${ }^{4} \mathrm{D}_{1 / 2}$ & ${ }^{2} \mathrm{P}_{1 / 2}$ & $2 \mathrm{~s}^{2} 2 \mathrm{p}^{4}\left({ }^{3} \mathrm{P}\right) 3 \mathrm{~d}^{1}$ \\
\hline 46 & ${ }^{4} \mathrm{D}_{3 / 2}$ & ${ }^{2} \mathrm{D}_{3 / 2}$ & $2 s^{2} 2 p^{4}\left({ }^{3} P\right) 3 d^{1}$ \\
\hline 74 & ${ }^{4} \mathrm{D}_{5 / 2}$ & ${ }^{4} \mathrm{P}_{5 / 2}$ & $2 s^{1} 2 p^{5}\left({ }^{3} P\right) 3 p^{1}$ \\
\hline 94 & ${ }^{2} \mathrm{~F}_{5 / 2}$ & ${ }^{2} \mathrm{D}_{5 / 2}$ & $2 s^{1} 2 p^{5}\left({ }^{3} P\right) 3 d^{1}$ \\
\hline 95 & ${ }^{2} \mathrm{~F}_{7 / 2}$ & ${ }^{4} \mathrm{D}_{7 / 2}$ & $2 s^{1} 2 p^{5}\left({ }^{3} P\right) 3 d^{1}$ \\
\hline 138 & ${ }^{2} \mathrm{D}_{5 / 2}$ & ${ }^{2} \mathrm{~F}_{5 / 2}$ & $2 s^{2} 2 p^{4}\left({ }^{3} P\right) 4 d^{1}$ \\
\hline 152 & ${ }^{4} \mathrm{~F}_{3 / 2}$ & ${ }^{4} \mathrm{D}_{3 / 2}$ & $2 \mathrm{~s}^{2} 2 \mathrm{p}^{4}\left({ }^{3} \mathrm{P}\right) 4 \mathrm{f}^{1}$ \\
\hline 153 & ${ }^{4} \mathrm{D}_{5 / 2}$ & ${ }^{2} \mathrm{~F}_{5 / 2}$ & $2 \mathrm{~s}^{2} 2 \mathrm{p}^{4}\left({ }^{3} \mathrm{P}\right) 4 \mathrm{f}^{1}$ \\
\hline 161 & ${ }^{4} \mathrm{D}_{3 / 2}$ & ${ }^{4} \mathrm{P}_{3 / 2}$ & $2 s^{2} 2 p^{4}\left({ }^{3} P\right) 4 d^{1}$ \\
\hline 167 & ${ }^{4} \mathrm{G}_{7 / 2}$ & ${ }^{2} \mathrm{G}_{7 / 2}$ & $2 \mathrm{~s}^{2} 2 \mathrm{p}^{4}\left({ }^{3} \mathrm{P}\right) 4 \mathrm{f}^{1}$ \\
\hline 207 & ${ }^{4} \mathrm{D}_{5 / 2}$ & ${ }^{4} \mathrm{P}_{5 / 2}$ & $2 \mathrm{~s}^{2} 2 \mathrm{p}^{4}\left({ }^{3} \mathrm{P}\right) 5 \mathrm{p}^{1}$ \\
\hline 216 & ${ }^{4} \mathrm{D}_{5 / 2}$ & ${ }^{4} \mathrm{P}_{5 / 2}$ & $2 s^{1} 2 p^{5}\left({ }^{3} P\right) 4 p^{1}$ \\
\hline 229 & ${ }^{2} \mathrm{D}_{3 / 2}$ & ${ }^{2} \mathrm{P}_{3 / 2}$ & $2 \mathrm{~s}^{2} 2 \mathrm{p}^{4}\left({ }^{3} \mathrm{P}\right) 5 \mathrm{~d}^{1}$ \\
\hline 246 & ${ }^{4} \mathrm{D}_{3 / 2}$ & ${ }^{2} \mathrm{D}_{3 / 2}$ & $2 \mathrm{~s}^{2} 2 \mathrm{p}^{4}\left({ }^{3} \mathrm{P}\right) 5 \mathrm{f}^{1}$ \\
\hline 270 & ${ }^{4} \mathrm{D}_{3 / 2}$ & ${ }^{4} \mathrm{P}_{3 / 2}$ & $2 \mathrm{~s}^{2} 2 \mathrm{p}^{4}\left({ }^{3} \mathrm{P}\right) 5 \mathrm{~d}^{1}$ \\
\hline 273 & ${ }^{2} \mathrm{P}_{3 / 2}$ & ${ }^{4} \mathrm{P}_{3 / 2}$ & $2 s^{1} 2 p^{5}\left({ }^{3} P\right) 4 p^{1}$ \\
\hline 275 & ${ }^{2} \mathrm{D}_{5 / 2}$ & ${ }^{2} \mathrm{~F}_{5 / 2}$ & $2 \mathrm{~s}^{2} 2 \mathrm{p}^{4}\left({ }^{3} \mathrm{P}\right) 5 \mathrm{~d}^{1}$ \\
\hline 278 & ${ }^{4} D_{5 / 2}$ & ${ }^{4} \mathrm{P}_{5 / 2}$ & $2 s^{1} 2 p^{5}\left({ }^{3} P\right) 4 d^{1}$ \\
\hline 287 & ${ }^{4} \mathrm{H}_{7 / 2}$ & ${ }^{2} \mathrm{~F}_{7 / 2}$ & $2 \mathrm{~s}^{2} 2 \mathrm{p}^{4}\left({ }^{3} \mathrm{P}\right) 5 \mathrm{~g}^{1}$ \\
\hline 303 & ${ }^{4} \mathrm{~F}_{9 / 2}$ & ${ }^{2} \mathrm{H}_{9 / 2}$ & $2 \mathrm{~s}^{2} 2 \mathrm{p}^{4}\left({ }^{3} \mathrm{P}\right) 5 \mathrm{~g}^{1}$ \\
\hline 316 & ${ }^{2} \mathrm{~F}_{5 / 2}$ & ${ }^{2} \mathrm{D}_{5 / 2}$ & $2 \mathrm{~s}^{1} 2 \mathrm{p}^{5}\left({ }^{3} \mathrm{P}\right) 4 \mathrm{f}^{1}$ \\
\hline 319 & ${ }^{4} \mathrm{~F}_{7 / 2}$ & ${ }^{4} \mathrm{D}_{7 / 2}$ & $2 s^{1} 2 p^{5}\left({ }^{3} P\right) 4 d^{1}$ \\
\hline 322 & ${ }^{2} \mathrm{~F}_{5 / 2}$ & ${ }^{4} \mathrm{D}_{5 / 2}$ & $2 s^{1} 2 p^{5}\left({ }^{3} P\right) 4 d^{1}$ \\
\hline 356 & ${ }^{2} \mathrm{~F}_{7 / 2}$ & ${ }^{4} \mathrm{G}_{7 / 2}$ & $2 \mathrm{~s}^{1} 2 \mathrm{p}^{5}\left({ }^{3} \mathrm{P}\right) 4 \mathrm{f}^{1}$ \\
\hline 357 & ${ }^{4} \mathrm{~F}_{9 / 2}$ & ${ }^{4} \mathrm{G}_{9 / 2}$ & $2 s^{1} 2 p^{5}\left({ }^{3} P\right) 4 f^{1}$ \\
\hline 363 & ${ }^{4} \mathrm{G}_{5 / 2}$ & ${ }^{4} \mathrm{~F}_{5 / 2}$ & $2 s^{1} 2 p^{5}\left({ }^{3} P\right) 4 f^{1}$ \\
\hline 364 & ${ }^{4} \mathrm{~F}_{7 / 2}$ & ${ }^{2} \mathrm{G}_{7 / 2}$ & $2 s^{1} 2 p^{5}\left({ }^{3} P\right) 4 f^{1}$ \\
\hline
\end{tabular}

numerics. However, they are not very important. The discrepancy between two forms with $f \geq 0.1$ (498 E1 transitions) does 
Table 3. Comparison of some calculated $\left(E^{\mathrm{GRASP}}\right)$ Fe XVIII energy levels with data presented by NIST $\left(E^{\mathrm{NIST}}\right)$.

\begin{tabular}{|c|c|c|c|c|c|c|c|c|c|}
\hline Index & Configuration & LSJ & $E^{\text {GRASP }}\left(\mathrm{cm}^{-1}\right)$ & $E^{\mathrm{NIST}}\left(\mathrm{cm}^{-1}\right)$ & Index & Configuration & LSJ & $E^{\text {GRASP }}\left(\mathrm{cm}^{-1}\right)$ & $E^{E^{\mathrm{NIST}}\left(\mathrm{cm}^{-1}\right)}$ \\
\hline 1 & $2 s^{2} 2 p^{5}$ & ${ }^{2} \mathrm{P}_{3 / 2}$ & 0. & 0. & 75 & $2 s^{1} 2 p^{5}\left({ }^{3} P\right) 3 p^{1}$ & ${ }^{4} \mathrm{P}_{3 / 2}$ & 7510542. & 7529900. \\
\hline 2 & $2 s^{2} 2 p^{5}$ & ${ }^{2} \mathrm{P}_{1 / 2}$ & 102063. & 102579. & 80 & $2 s^{1} 2 p^{5}\left({ }^{3} P\right) 3 p^{1}$ & ${ }^{2} S_{1 / 2}$ & 7582841. & 7599400. \\
\hline 3 & $2 s^{1} 2 p^{6}$ & ${ }^{2} \mathrm{~S}_{1 / 2}$ & 1079594. & 1064702. & 87 & $2 \mathrm{~s}^{1} 2 \mathrm{p}^{5}\left({ }^{1} \mathrm{P}\right) 3 \mathrm{p}^{1}$ & ${ }^{2} \mathrm{D}_{3 / 2}$ & 7770006. & 7763400 . \\
\hline 4 & $2 s^{2} 2 p^{4}\left({ }^{3} P\right) 3 s^{1}$ & ${ }^{4} \mathrm{P}_{5 / 2}$ & 6206772. & 6222000. & 90 & $2 s^{1} 2 p^{5}\left({ }^{1} P\right) 3 p^{1}$ & ${ }^{2} \mathrm{D}_{5 / 2}$ & 7792996. & 7783900. \\
\hline 5 & $2 s^{2} 2 p^{4}\left({ }^{3} P\right) 3 s^{1}$ & ${ }^{2} \mathrm{P}_{3 / 2}$ & 6234827. & 6248100. & 91 & $2 s^{1} 2 p^{5}\left({ }^{1} P\right) 3 p^{1}$ & ${ }^{2} \mathrm{P}_{1 / 2}$ & 7802019. & 7786000. \\
\hline 6 & $2 s^{2} 2 p^{4}\left({ }^{3} P\right) 3 s^{1}$ & ${ }^{4} \mathrm{P}_{1 / 2}$ & 6285591. & 6310200. & 92 & $2 s^{1} 2 p^{5}\left({ }^{1} P\right) 3 p^{1}$ & ${ }^{2} \mathrm{P}_{3 / 2}$ & 7812627. & 7794400 . \\
\hline 7 & $2 s^{2} 2 p^{4}\left({ }^{3} P\right) 3 s^{1}$ & ${ }^{4} \mathrm{P}_{3 / 2}$ & 6303529. & 6317900. & 110 & $2 s^{2} 2 p^{4}\left({ }^{3} P\right) 4 s^{1}$ & ${ }^{2} \mathrm{P}_{3 / 2}$ & 8416632. & 8428200 . \\
\hline 8 & $2 s^{2} 2 p^{4}\left({ }^{3} P\right) 3 s^{1}$ & ${ }^{2} \mathrm{P}_{1 / 2}$ & 6329621. & 6342600. & 113 & $2 s^{2} 2 p^{4}\left({ }^{3} P\right) 4 s^{1}$ & ${ }^{4} \mathrm{P}_{3 / 2}$ & 8497775 . & 8517200 . \\
\hline 9 & $2 s^{2} 2 p^{4}\left({ }^{1} D\right) 3 s^{1}$ & ${ }^{2} \mathrm{D}_{5 / 2}$ & 6387985. & 6400000. & 121 & $2 s^{2} 2 p^{4}\left({ }^{1} D\right) 4 s^{1}$ & ${ }^{2} \mathrm{D}_{5 / 2}$ & 8581039. & 8591100. \\
\hline 10 & $2 s^{2} 2 p^{4}\left({ }^{1} D\right) 3 s^{1}$ & ${ }^{2} \mathrm{D}_{3 / 2}$ & 6391884. & 6403800. & 122 & $2 s^{2} 2 p^{4}\left({ }^{1} D\right) 4 s^{1}$ & ${ }^{2} \mathrm{D}_{3 / 2}$ & 8582291. & 8593000 . \\
\hline 18 & $2 s^{2} 2 p^{4}\left({ }^{1} S\right) 3 s^{1}$ & ${ }^{2} \mathrm{~S}_{1 / 2}$ & 6543747. & 6575100. & 137 & $2 \mathrm{~s}^{2} 2 \mathrm{p}^{4}\left({ }^{3} \mathrm{P}\right) 4 \mathrm{~d}^{1}$ & ${ }^{2} \mathrm{D}_{3 / 2}$ & 8664575. & 8676000. \\
\hline 38 & $2 s^{2} 2 p^{4}\left({ }^{3} P\right) 3 d^{1}$ & ${ }^{4} \mathrm{P}_{1 / 2}$ & 6845037. & 6858200. & 138 & $2 s^{2} 2 p^{4}\left({ }^{3} P\right) 4 d^{1}$ & ${ }^{2} \mathrm{D}_{5 / 2}$ & 8666954. & 8676000 . \\
\hline 40 & $2 s^{2} 2 p^{4}\left({ }^{3} P\right) 3 d^{1}$ & ${ }^{4} \mathrm{P}_{3 / 2}$ & 6860872. & 6872400. & 156 & $2 s^{2} 2 p^{4}\left({ }^{3} P\right) 4 d^{1}$ & ${ }^{4} \mathrm{~F}_{3 / 2}$ & 8720942. & 8727500 . \\
\hline 41 & $2 s^{2} 2 p^{4}\left({ }^{3} P\right) 3 d^{1}$ & ${ }^{2} \mathrm{~F}_{5 / 2}$ & 6869054. & 6880400. & 158 & $2 \mathrm{~s}^{2} 2 \mathrm{p}^{4}\left({ }^{3} \mathrm{P}\right) 4 \mathrm{~d}^{1}$ & ${ }^{4} \mathrm{~F}_{5 / 2}$ & 8722504. & 8727500 . \\
\hline 42 & $2 s^{2} 2 p^{4}\left({ }^{3} P\right) 3 d^{1}$ & ${ }^{4} \mathrm{D}_{1 / 2}$ & 6886694. & 6903200. & 162 & $2 s^{2} 2 p^{4}\left({ }^{3} P\right) 4 d^{1}$ & ${ }^{4} \mathrm{P}_{5 / 2}$ & 8736284. & 8756600 . \\
\hline 46 & $2 s^{2} 2 p^{4}\left({ }^{3} P\right) 3 d^{1}$ & ${ }^{4} \mathrm{D}_{3 / 2}$ & 6906682. & 6919000. & 164 & $2 \mathrm{~s}^{2} 2 \mathrm{p}^{4}\left({ }^{3} \mathrm{P}\right) 4 \mathrm{~d}^{1}$ & ${ }^{2} \mathrm{P}_{3 / 2}$ & 8750241. & 8759900. \\
\hline 47 & $2 s^{2} 2 p^{4}\left({ }^{3} P\right) 3 d^{1}$ & ${ }^{4} \mathrm{P}_{5 / 2}$ & 6922310. & 6903700. & 176 & $2 \mathrm{~s}^{2} 2 \mathrm{p}^{4}\left({ }^{1} \mathrm{D}\right) 4 \mathrm{~d}^{1}$ & ${ }^{2} \mathrm{D}_{5 / 2}$ & 8817821. & 8829200. \\
\hline 48 & $2 s^{2} 2 p^{4}\left({ }^{3} P\right) 3 d^{1}$ & ${ }^{2} \mathrm{P}_{3 / 2}$ & 6935515. & 6947300. & 177 & $2 s^{2} 2 p^{4}\left({ }^{1} D\right) 4 d^{1}$ & ${ }^{2} \mathrm{~S}_{1 / 2}$ & 8819585. & 8829200. \\
\hline 49 & $2 s^{2} 2 p^{4}\left({ }^{3} P\right) 3 d^{1}$ & ${ }^{2} D_{5 / 2}$ & 6947225. & 6957000. & 178 & $2 \mathrm{~s}^{2} 2 \mathrm{p}^{4}\left({ }^{1} \mathrm{D}\right) 4 \mathrm{~d}^{1}$ & ${ }^{2} \mathrm{P}_{3 / 2}$ & 8820801. & 8829200. \\
\hline 53 & $2 \mathrm{~s}^{2} 2 \mathrm{p}^{4}\left({ }^{1} \mathrm{D}\right) 3 \mathrm{~d}^{1}$ & ${ }^{2} S_{1 / 2}$ & 7005754 . & 7014300. & 180 & $2 \mathrm{~s}^{2} 2 \mathrm{p}^{4}\left({ }^{1} \mathrm{D}\right) 4 \mathrm{~d}^{1}$ & ${ }^{2} \mathrm{~F}_{5 / 2}$ & 8824274. & 8829200. \\
\hline 55 & $2 \mathrm{~s}^{2} 2 \mathrm{p}^{4}\left({ }^{1} \mathrm{D}\right) 3 \mathrm{~d}^{1}$ & ${ }^{2} \mathrm{P}_{3 / 2}$ & 7033450 . & 7038400 . & 181 & $2 \mathrm{~s}^{2} 2 \mathrm{p}^{4}\left({ }^{1} \mathrm{D}\right) 4 \mathrm{~d}^{1}$ & ${ }^{2} \mathrm{D}_{3 / 2}$ & 8834034. & 8843900 . \\
\hline 56 & $2 \mathrm{~s}^{2} 2 \mathrm{p}^{4}\left({ }^{1} \mathrm{D}\right) 3 \mathrm{~d}^{1}$ & ${ }^{2} \mathrm{D}_{5 / 2}$ & 7035986. & 7040800. & 182 & $2 \mathrm{~s}^{2} 2 \mathrm{p}^{4}\left({ }^{1} \mathrm{D}\right) 4 \mathrm{~d}^{1}$ & ${ }^{2} \mathrm{P}_{1 / 2}$ & 8837105. & 8843900 . \\
\hline 57 & $2 \mathrm{~s}^{2} 2 \mathrm{p}^{4}\left({ }^{1} \mathrm{D}\right) 3 \mathrm{~d}^{1}$ & ${ }^{2} \mathrm{D}_{3 / 2}$ & 7060411. & 7066200. & 196 & $2 s^{2} 2 p^{4}\left({ }^{1} S\right) 4 d^{1}$ & ${ }^{2} \mathrm{D}_{3 / 2}$ & 8999740. & 8989200. \\
\hline 58 & $2 \mathrm{~s}^{2} 2 \mathrm{p}^{4}\left({ }^{1} \mathrm{D}\right) 3 \mathrm{~d}^{1}$ & ${ }^{2} \mathrm{P}_{1 / 2}$ & 7070305. & 7074200 . & 231 & $2 \mathrm{~s}^{2} 2 \mathrm{p}^{4}\left({ }^{3} \mathrm{P}\right) 5 \mathrm{~d}^{1}$ & ${ }^{2} \mathrm{D}_{5 / 2}$ & 9488436. & 9510000. \\
\hline 59 & $2 s^{2} 2 p^{4}\left({ }^{1} S\right) 3 d^{1}$ & ${ }^{2} \mathrm{D}_{5 / 2}$ & 7153608. & 7166400. & 275 & $2 \mathrm{~s}^{2} 2 \mathrm{p}^{4}\left({ }^{3} \mathrm{P}\right) 5 \mathrm{~d}^{1}$ & ${ }^{2} \mathrm{D}_{5 / 2}$ & 9570721. & 9610000. \\
\hline 61 & $2 s^{2} 2 p^{4}\left({ }^{1} S\right) 3 d^{1}$ & ${ }^{2} \mathrm{D}_{3 / 2}$ & 7175002. & 7184300. & 279 & $2 \mathrm{~s}^{2} 2 \mathrm{p}^{4}\left({ }^{3} \mathrm{P}\right) 5 \mathrm{~d}^{1}$ & ${ }^{2} \mathrm{D}_{3 / 2}$ & 9575847. & 9640000. \\
\hline 69 & $2 s^{1} 2 p^{5}\left({ }^{3} P\right) 3 p^{1}$ & ${ }^{4} \mathrm{D}_{3 / 2}$ & 7446302. & 7464400. & 325 & $2 \mathrm{~s}^{2} 2 \mathrm{p}^{4}\left({ }^{1} \mathrm{D}\right) 5 \mathrm{~d}^{1}$ & ${ }^{2} \mathrm{P}_{3 / 2}$ & 9648395. & 9680000. \\
\hline 72 & $2 s^{1} 2 p^{5}\left({ }^{3} P\right) 3 p^{1}$ & ${ }^{2} \mathrm{P}_{3 / 2}$ & 7486040. & 7487800. & 327 & $2 \mathrm{~s}^{2} 2 \mathrm{p}^{4}\left({ }^{1} \mathrm{D}\right) 5 \mathrm{~d}^{1}$ & ${ }^{2} \mathrm{~F}_{5 / 2}$ & 9650514. & 9680000. \\
\hline 73 & $2 s^{1} 2 p^{5}\left({ }^{3} P\right) 3 p^{1}$ & ${ }^{2} \mathrm{P}_{1 / 2}$ & 7504936. & 7508100. & 329 & $2 \mathrm{~s}^{2} 2 \mathrm{p}^{4}\left({ }^{1} \mathrm{D}\right) 5 \mathrm{~d}^{1}$ & ${ }^{2} \mathrm{D}_{3 / 2}$ & 9654660. & 9680000. \\
\hline 74 & $2 s^{1} 2 p^{5}\left({ }^{3} P\right) 3 p^{1}$ & ${ }^{4} \mathrm{D}_{5 / 2}$ & 7505906. & 7508100. & 331 & $2 \mathrm{~s}^{2} 2 \mathrm{p}^{4}\left({ }^{1} \mathrm{D}\right) 5 \mathrm{~d}^{1}$ & ${ }^{2} \mathrm{P}_{1 / 2}$ & 9656026. & 9680000. \\
\hline
\end{tabular}

Table 4. Comparison of some calculated (GRASP, Cornille et al. 1992 and Sampson et al. 1991) Fe XVIII radiative transition probabilities $A^{r}$ (in $\mathrm{s}^{-1}$ ) with data presented by NIST. Note that $a(b) \equiv a \times 10^{b}$.

\begin{tabular}{rrrrrrr}
\hline \hline$i$ & $j$ & Transition & NIST & GRASP & Cornille & Sampson \\
\hline 1 & $2 \mathrm{M} 1$ & ${ }^{2} \mathrm{P}_{3 / 2}-{ }^{2} \mathrm{P}_{1 / 2}$ & $1.94(04)$ & $1.905(04)$ & & \\
1 & $2 \mathrm{E} 2$ & ${ }^{2} \mathrm{P}_{3 / 2}-{ }^{2} \mathrm{P}_{1 / 2}$ & $1.90(00)$ & $1.941(00)$ & & \\
1 & 3 & ${ }^{2} \mathrm{P}_{3 / 2}-{ }^{2} \mathrm{~S}_{1 / 2}$ & $9.13(10)$ & $8.313(10)$ & $8.575(10)$ & $9.501(10)$ \\
1 & 4 & ${ }^{2} \mathrm{P}_{3 / 2}-{ }^{4} \mathrm{P}_{5 / 2}$ & $9.10(10)$ & $8.312(10)$ & $8.765(10)$ & $7.385(10)$ \\
1 & 6 & ${ }^{2} \mathrm{P}_{3 / 2}-{ }^{4} \mathrm{P}_{1 / 2}$ & $2.00(11)$ & $1.703(11)$ & $1.275(11)$ & $1.796(11)$ \\
1 & 8 & ${ }^{2} \mathrm{P}_{3 / 2}-{ }^{2} \mathrm{P}_{1 / 2}$ & $1.40(12)$ & $1.234(12)$ & $1.213(12)$ & $1.233(12)$ \\
1 & 9 & ${ }^{2} \mathrm{P}_{3 / 2}-{ }^{2} \mathrm{D}_{5 / 2}$ & $1.10(12)$ & $1.021(12)$ & $9.617(11)$ & $8.911(11)$ \\
1 & 18 & ${ }^{2} \mathrm{P}_{3 / 2}-{ }^{2} \mathrm{~S}_{1 / 2}$ & $2.80(11)$ & $2.285(11)$ & $3.321(11)$ & $2.230(11)$ \\
1 & 53 & ${ }^{2} \mathrm{P}_{3 / 2}-{ }^{2} \mathrm{~S}_{1 / 2}$ & $1.60(13)$ & $1.474(13)$ & $1.504(13)$ & $1.323(13)$ \\
1 & 55 & ${ }^{2} \mathrm{P}_{3 / 2}-{ }^{2} \mathrm{P}_{3 / 2}$ & $1.90(13)$ & $1.929(13)$ & $2.111(13)$ & $1.900(13)$ \\
1 & 57 & ${ }^{2} \mathrm{P}_{3 / 2}-{ }^{2} \mathrm{D}_{3 / 2}$ & $4.30(12)$ & $3.944(12)$ & $3.525(12)$ & $4.346(12)$ \\
1 & 59 & ${ }^{2} \mathrm{P}_{3 / 2}-{ }^{2} \mathrm{D}_{5 / 2}$ & $1.10(12)$ & $1.152(12)$ & $1.722(12)$ & $1.114(12)$ \\
1 & 61 & ${ }^{2} \mathrm{P}_{3 / 2}-{ }^{2} \mathrm{D}_{3 / 2}$ & $9.60(10)$ & $6.240(10)$ & $1.466(11)$ & $8.594(10)$ \\
2 & 3 & ${ }^{2} \mathrm{P}_{1 / 2}-{ }^{2} \mathrm{~S}_{1 / 2}$ & $3.31(10)$ & $3.035(10)$ & $3.144(10)$ & $3.471(10)$ \\
2 & 8 & ${ }^{2} \mathrm{P}_{1 / 2}-{ }^{2} \mathrm{P}_{1 / 2}$ & $1.50(12)$ & $1.399(12)$ & $1.407(12)$ & $1.183(12)$ \\
2 & 10 & ${ }^{2} \mathrm{P}_{1 / 2}-{ }^{2} \mathrm{D}_{3 / 2}$ & $1.30(12)$ & $1.240(12)$ & $1.228(12)$ & $1.088(12)$ \\
2 & 18 & ${ }^{2} \mathrm{P}_{1 / 2}-{ }^{2} \mathrm{~S}_{1 / 2}$ & $1.10(12)$ & $8.909(11)$ & $8.613(11)$ & $8.423(11)$ \\
2 & 53 & ${ }^{2} \mathrm{P}_{1 / 2}-{ }^{2} \mathrm{~S}_{1 / 2}$ & $2.70(12)$ & $2.812(12)$ & $2.538(12)$ & $2.624(12)$ \\
2 & 55 & ${ }^{2} \mathrm{P}_{1 / 2}-{ }^{2} \mathrm{P}_{3 / 2}$ & $3.20(12)$ & $2.838(12)$ & $3.008(12)$ & $2.915(12)$ \\
2 & 57 & ${ }^{2} \mathrm{P}_{1 / 2}{ }^{2} \mathrm{D}_{3 / 2}$ & $1.50(13)$ & $1.333(13)$ & $1.564(13)$ & $1.313(13)$ \\
2 & 61 & ${ }^{2} \mathrm{P}_{1 / 2}-{ }^{2} \mathrm{D}_{3 / 2}$ & $1.50(13)$ & $1.436(13)$ & $1.390(13)$ & $1.548(13)$ \\
\hline
\end{tabular}


not exceed $20 \%$ for most oscillator strengths, and only 13 transitions have larger deviation but which is still less than $60 \%$. The average deviation is $4.7 \%$ for $f \geq 0.1$ and $9.5 \%$ for $f \geq 0.01$ (2483 transitions). This is highly satisfactory for large calculations. For E2 transitions with $f \geq 10^{-5}$, only 4 oscillator strengths of the two forms have differences exceeding $20 \%$, but are still less than $30 \%$. The average of deviation is $1.9 \%$ (767 transitions).

The largest contribution of forbidden E2 and M1 transitions to the total radiative transition probability is to levels of the $1 s^{2} 2 s^{2} 2 p^{4} 3 p$ configuration. It exceeds $10 \%$ of the total radiative transition probability for 8 levels, 7 of which arise from this configuration. The contribution of forbidden transitions is largest $(26 \%)$ for level $15\left(2 \mathrm{p}_{1 / 2}^{2} 2 \mathrm{p}_{3 / 2}^{2} 3 \mathrm{p}_{3 / 2}^{1}{ }^{2} \mathrm{D}_{5 / 2}\right)$. For level $25\left(2 \mathrm{p}_{1 / 2}^{1} 2 \mathrm{p}_{3 / 2}^{3} 3 \mathrm{p}_{1 / 2}^{1}{ }^{2} \mathrm{~F}_{5 / 2}\right)$ the inclusion of these type of transitions corresponds to $21 \%$ of the total radiative transition probability. The contribution of E3 and M2 transitions, which are not presented here, is less than $0.1 \%$.

\section{Conclusion}

In this paper we have presented results for the 379 lowest energy levels of the $1 \mathrm{~s}^{2} 2 \mathrm{~s}^{2} 2 \mathrm{p}^{5}, \quad 1 \mathrm{~s}^{2} 2 \mathrm{~s}^{1} 2 \mathrm{p}^{6}, \quad 1 \mathrm{~s}^{2} 2 \mathrm{~s}^{2} 2 \mathrm{p}^{4} 3 l$, $1 \mathrm{~s}^{2} 2 \mathrm{~s}^{2} 2 \mathrm{p}^{4} 4 l^{\prime}, \quad 1 \mathrm{~s}^{2} 2 \mathrm{~s}^{2} 2 \mathrm{p}^{4} 5 l^{\prime \prime}, \quad 1 \mathrm{~s}^{2} 2 \mathrm{p}^{6} 3 l, \quad 1 \mathrm{~s}^{2} 2 \mathrm{~s}^{1} 2 \mathrm{p}^{5} 3 l$, $1 \mathrm{~s}^{2} 2 \mathrm{~s}^{1} 2 \mathrm{p}^{5} 4 l^{\prime}$ and $1 \mathrm{~s}^{2} 2 \mathrm{~s}^{2} 2 \mathrm{p}^{4} 6 \mathrm{~s}\left(l=0,1,2, l^{\prime}=0,1,2,3\right.$ and $\left.l^{\prime \prime}=0,1,2,3,4\right)$ configurations of fluorine-like Fe XVIII, calculated in the multiconfigurational Dirac-Fock approximation with the GRASP code. To our knowledge, this is the largest calculation for Fe XVIII to date. Characteristics of levels in the LS-coupling scheme have been provided, and checked for their completeness. Transition probabilities for E1, E2 and M1 transitions among the 379 levels have also been calculated. The energy level positions agree with experimental values within $0.7 \%$, and in most cases even better. The only exception is a $1.4 \%$ deviation for the energy of the $1 s^{2} 2 s^{1} 2 p^{6}{ }^{2} S_{1 / 2}$ level. Differences between the calculated transition probabilities and experiment are largest $(35 \%)$ for the weaker transitions from levels of the $1 s^{2} 2 s^{2} 2 p_{3 / 2}^{4} 3 d_{3 / 2}$ configuration to the ground state, while for other available experimental data agreement is within $20 \%$ and in most cases far better.

The total radiative transition probability and two strongest probabilities from each level have been calculated, taking into account forbidden transitions. Contributions of forbidden E2 and M1 transitions to the lifetimes of levels does not exceed $26 \%$.

Based on the complexity of our theoretical results for Fe XVIII obtained the using relativistic multiconfigurational Dirac-Fock approach, and their agreement with the available experimental values, we conclude that our calculations of energy levels and transition data are reliable. They may hence be confidently applied to the interpretation of astronomical and other spectral observations. For example, absorption lines of Fe XVIII have been detected in XMM-Newton and Chandra spectra of active galaxies (Dubau et al. 2003), while many transitions have been detected in emission from the Sun (Cornille et al. 1992). Analysis of these observations using our new atomic data should allow more reliable models of the Fe XVIII absorbing or emitting plasma to be derived. For example, in solar spectra the Fe XVIII emission lines provide a valuable temperature diagnostic (Cornille et al. 1994). We hope to undertake such work in the future.

Acknowledgements. FPK is grateful to AWE Aldermaston for the award of a William Penney Fellowship. This work was supported by PPARC and EPSRC, and also by NATO Collaborative Linkage Grant CLG.979443. We are also grateful to the Defence Science and Technology Laboratory (dstl) for support under the Joint Grants Scheme.

\section{References}

Armstrong, Jr. L. 1966, J. Math. Phys., 7, 1891

Badnell, N. R. 1986, J. Phys. B, 19, 3827

Badnell, N. R. 1997, J. Phys. B, 30, 1

Blackford, H. M. S., \& Hibbert, A. 1994, At. Data Nucl. Data Tables, 58,101

Bromage, G. E., Fawcett, B. C., \& Cowan, R. D. 1977, MNRAS, 178, 599

Burke, P. G., Hibbert, A., \& Robb, W. D. 1971, J. Phys. B, 4, 153

Cornille, M., Dubau, J., Loulergue, M., Bely-Dubau, F., \& Faucher, P. 1992, A\&A, 259, 669

Cornille, M., Dubau, J., Faucher, P., Bely-Dubau, F., \& Blancard, C. 1994, A\&AS, 105, 77

Cowan, R. D. 1981, The Theory of Atomic Structure and Spectra (Berkeley, CA: University of California Press)

Dubau, J., Porquet, D., \& Zabaydullin, O. Z. 2003, J. Quant. Spec. Radiat. Transf., 81, 117

Dyall, K. G., Grant, I. P., Johnson, C. T., Parpia, F. A., \& Plummer, E. P. 1989, Comput. Phys. Comm., 35, 425

Eissner, W., Jones, M., \& Nussbaumer, H. 1974, Comput. Phys. Commun., 8, 270

Fawcett, B. C. 1984, At. Data Nucl. Data Tables, 31, 495

Hibbert, A., \& Glass, R. 1978, Comput. Phys. Commun., 16, 19

Karazija, R., \& Jonauskas, V. 2001, J. Math. Phys., 42, 5642

Palmeri, P., Mendoza, C., Kallman, T. R., \& Bautista, M. A. 2003, A\&A, 403, 1175

Sampson, D. H., Zhang, H. L., \& Fontes, C. J. 1991, At. Data Nucl. Data Tables, 48, 25 